\title{
Health inequalities among students of lower secondary schools in Bytom, Poland
}

\author{
Agata Wypych-Ślusarska', Elżbieta Czech², Joanna Kasznia-Kocot' ${ }^{1}$, Jerzy Słowiński ${ }^{1}$, \\ Ewa Niewiadomska ${ }^{2}$, Michał Skrzypek ${ }^{2}$, Jolanta Malinowska-Borowska ${ }^{3}$ \\ ${ }^{1}$ Medical University of Silesia, Katowice, School of Public Health in Bytom, Department of Epidemiology, Bytom, Poland \\ ${ }^{2}$ Medical University of Silesia, Katowice, School of Public Health in Bytom, Department of Biostatistics, Bytom, Poland \\ ${ }^{3}$ Medical University of Silesia, Katowice, School of Public Health in Bytom, Department of Toxicology and Occupational \\ Health Protection, Bytom, Poland
}

Wypych-Ślusarska A, Czech E, Kasznia-Kocot J, Słowiński J, Niewiadomska E, Skrzypek M, Malinowska-Borowska J. Health inequalities among students of lower secondary schools in Bytom, Poland. Ann Agric Environ Med. 2018; 25(1): 4-8. doi: 10.5604/12321966.1232771

\section{Abstract}

Introduction and objective. Poverty and low level of education pose the biggest threats to public health. Moreover, they generate inequalities in public healthThe aim of the study was to check if there are any inequalities in health among teenagers living in Bytom, Poland.

Materials and method. In 2011 and 2012, an epidemiological cross-sectional study was conducted on 1,099 students from lower secondary schools from Bytom. The students completed a questionnaire which was based on an earlier Health Behaviour in School-aged Children study (HBSC). Socio-Economic Status of teenagers (SES) was determined according to the Family Affluence Scale (FAS), the intensity of possible problems in the place of residence and on parents' education. Impact of SES on health self-assessment, asthma, pneumonia, bronchitis with addition to spinal deformities were also investigated. Results. A good or very good level of health was declared by students from families representing a high level of FAS and residing in a more peaceful, less troubled neighbourhood. The highest level of asthma prevalence (10.9\%) occurred among students from families with a low level of FAS. The students from families with high FAS were less affected by spinal deformities (34.6\%). Students living in a troubled neighbourhood more often suffered from bronchial asthma, pneumonia, bronchitis and spinal deformities.

Conclusions. The level of family affluence depends on the parents' education and all the analysed health problems occurred more frequently in children whose parents had completed at least general secondary education. A high economic standard of living and a peaceful neighbourhood determined good or very good health self-assessment among the surveyed students.

\section{Key words}

health inequalities, socio-economic status, teenagers

\section{INTRODUCTION}

Health is a very important factor that determines social and economic development of the population. The existing conditions and differences in the state of health have become more visible and recognizable and constant striving for equality in health is one of the most important objectives for the health policy of the WHO and EU [1-2].

Any variability and differences in the state of health between individuals and groups of people are considered health inequalities. The WHO adopted the definition of health inequalities set forth by Margaret Whitehead, Prof. of Public Health at the Institute of Psychology, University of Liverpool in the UK, who claimed that 'health inequalities are not only unnecessary and avoidable, but also unfair and unjust [3]. Health inequalities affect mostly children and teenagers. The basic factors responsible for the occurrence of health inequalities are of a social and economic nature and include: level of education, income and employment. Poverty, being one of the main causes of inequalities, has an impact on reducing the chances of personal, social and emotional development, as well as quality of young people's future lives [4]. According to statistical data, in

Addrerss for correspondente: Agata Wypych-Ślusarska, Medical University of Silesia, Public Health Faculty, Department of Epidemiology, 41-902 Bytom, Poland E-mail: awypych@sum.edu.pl

Received: 17 Jule 2014; accepted: 9 March 2015; first published on February 2017
2010, approximately $12 \%$ of children and teenagers lived below the relative poverty threshold and over $8 \%$ lived below the absolute poverty threshold [5]. One of the main determinants of poverty is the level of unemployment. In January 2012, the unemployment level in Silesian Province in south-west Poland was $10.8 \%$, which was lower than the Polish average of $13.2 \%$ [6]. However, in the Silesian Province there is a huge diversity of unemployment. In Bytom district, the highest unemployment level was $15.6 \%$, in Bytom city centre, unemployment was at the highest level in the entire Silesian Province - 20\% [6]. Young people under the age of 34 constituted nearly half of the unemployed population. Another worrying fact was that $19.6 \%$ of all students rely on the free food subsidy provided by the State school system [7].

\section{OBJECTIVES}

At present worldwide, organisations dealing with public health are striving to diminish inequalities in health. In 1998, the World Health Declaration 'Health for All in the $21^{\text {st }}$ Century' was adopted. There was a plan to reduce differences in the state of health between social and economic groups of all the EU member states by at least $25 \%$ by the year 2020 . This objective could be realised by substantial health improvement of the underprivileged [8]. Bearing in mind all the above facts, this study was designed to investigate whether there 
are any inequalities in health among the students of lower secondary schools in Bytom.

\section{MATERIALS AND METHOD}

The epidemiological cross-sectional study was conducted at the turn of 2011 and 2012. A letter with an invitation to participate in the study was sent to headmasters of all lower-secondary schools in Bytom. Headmasters of schools declined to participate. Finally, questionnaires were handed out among 3,262 students, aged 13-16, from 11 lowersecondary schools in Bytom. Students who took part in the study had given their parents a letter explaining the reason and scope of this study, as well as declaration of consent to complete the questionnaire. The study resulted in 1,099 completed questionnaires $(33,7 \%)$. Of the total respondents, $55.6 \%$ were female and $44.4 \%$ male.

The survey was based on several questions originating from an earlier study, the Health Behaviour in School-aged Children (HBSC) [9]. The questionnaire issued included 64 questions concerning the social and economic status of each family, health condition, free time activities and taking stimulants.

Both social and economic status of each family was established according to the FAS Family Affluence Scale (FAS), the intensity of possible problems occurring in the place of residence, and parents' educational level. The FAS is based on 4 questions which estimate family wealth according to whether they own a family car, have a bedroom, travel with the family, and have a computer at home. The answer to each question is rated between $0-2$ points. The FAS scale ranges from $0-7$ points, $0-3=$ low, $4-5=$ medium, and $6-7$ a high level of FAS [10]. The scale of intensity of problems arising in the place of residence took into consideration the presence of troublemaking young people, rubbish and litter (i.e. broken glass), neglected homes and surrounding buildings. Students marked the intensity of these factors in a 3-point scale: a lot, little or none, and ranged from $0-6$ points, where 5-6 points meant a neighbourhood with the highest presence of the mentioned factors [10].

Analysis of relations between Socio-Economic Status (SES) and its influence on young people's health took into account health self-assessment, the occurrence of asthma, pneumonia, bronchitis and spinal deformities. Health selfassessment was based on the question: 'How would you rate your health?' The possible choices were: a) very good, b) good, c) moderate, d) bad, e) very bad.

Respondents were asked if they have been diagnosed with asthma, pneumonia, bronchitis and spinal deformities. The occurrence of asthma, pneumonia, bronchitis and spinal deformities were assessed according to this survey.

Statistical analysis. Qualitative data were summarized by absolute frequencies and percentages of its values. Statistical significance of differences between categorical variables was assessed by means of $\chi^{2}$ test. Statistical data analysis was conducted using Statistica 10.0 software (Statsoft 2010, Poland). The significance level was set at $\alpha=0.05$.

\section{RESULTS}

1,099 lower-secondary school students took part in the study (55.6\% females and $44.4 \%$ males). $39.5 \%$ of the interviewees represented medium and high levels of FAS, and every fifth respondent came from a family with low FAS (20.9\%). More than a half of the interviewed students (51.3\%) lived in a neighbourhood with a high intensity of problems, and $12.2 \%$ in a neighbourhood with a low intensity of problems. Approximately one-third of the respondents' parents had primary or vocational education: mothers $-29.9 \%$, fathers $-34.8 \%$.

Half of the students described their health as good (50.2\%) and one-third as very good (33.1\%). A characteristic fact was that the interviewed boys statistically self-assessed their health as better than girls $(41.3 \%$ vs. $26.6 \%$; $<<0.01)$. The most frequent health issues were: bronchitis (46.0\%), spinal deformities (38.0\%) and pneumonia (34.9\%). Bronchial asthma affected $9.7 \%$ of the children and occurred significantly more often in boys than girls $(12.1 \%$ vs. $7.9 \%$; $\mathrm{p}=0.02)$. Spinal deformities were declared significantly more often by girls than by the interviewed boys ( $43.4 \%$ vs. $31.2 \%$; $\mathrm{p}<0.01)$. While analysing both pneumonia and bronchitis, no gender differences were observed (35.1\% vs. $34.7 \%$ and $46.0 \%$ vs. $45.9 \%$, respectively). The socio-economic status and its influence on young people's health was also investigated and the detailed results are presented in Tables 1 and 2.

Table 1. Family Affluence Scale (FAS) vs. subjective assessment of health and health problems

\begin{tabular}{|c|c|c|c|c|}
\hline & \multicolumn{4}{|c|}{ FAS scale } \\
\hline & Low & Medium & High & $p$ value \\
\hline \multicolumn{5}{|c|}{ Health self-assessment ( $N=1078)$} \\
\hline Average or bad & $57(25.3 \%)$ & 68 (15.9\%) & 57 (13.4\%) & \multirow{3}{*}{$<0.001$} \\
\hline Rather good & $126(56.0 \%)$ & $216(50.6 \%)$ & $203(47.7 \%)$ & \\
\hline Very good & $42(18.7 \%)$ & $143(33.5 \%)$ & $166(39.0 \%)$ & \\
\hline Asthma ( $\mathrm{N}=1049)$ & 24 (10.9\%) & $38(9.1 \%)$ & $42(10.2 \%)$ & NS \\
\hline Pneumonia ( $N=1071)$ & $72(32.0 \%)$ & $163(38.5 \%)$ & $144(34.0 \%)$ & NS \\
\hline Bronchitis ( $N=1074)$ & $103(45.6 \%)$ & $204(48.1 \%)$ & $191(45.0 \%)$ & NS \\
\hline Spinal deformities ( $N=1069$ ) & $87(38.7 \%)$ & $174(41.2 \%)$ & $146(34.6 \%)$ & NS \\
\hline
\end{tabular}

NS - not significant

Table 2. Proliferation of problems in the place of living vs. subjective assessment of health and health problems

\begin{tabular}{|c|c|c|c|c|}
\hline & \multicolumn{4}{|c|}{ Proliferation of problems in the place of living } \\
\hline & Low & Average & High & $p$ value \\
\hline \multicolumn{5}{|c|}{ Health-self assessment ( $N=1083$ ) } \\
\hline Average or poor & $9(6.8 \%)$ & 49 (12.4\%) & $123(22.1 \%)$ & \multirow{3}{*}{$<0.001$} \\
\hline Rather good & $64(48.5 \%)$ & $218(55.2 \%)$ & $263(47.3 \%)$ & \\
\hline Very good & $59(44.7 \%)$ & $128(32.4 \%)$ & $170(30.6 \%)$ & \\
\hline Asthma ( $\mathrm{N}=1055)$ & $9(7.2 \%)$ & $39(10.0 \%)$ & $53(9.8 \%)$ & NS \\
\hline Pneumonia ( $\mathrm{N}=1076$ ) & $36(27.3 \%)$ & $128(32.7 \%)$ & $210(38.0 \%)$ & 0.04 \\
\hline Bronchitis ( $N=1079)$ & 47 (35.6\%) & 195 (49.7\%) & $252(45.4 \%)$ & 0.02 \\
\hline Spinal deformities ( $\mathrm{N}=1074)$ & 35 (27.1\%) & 155 (39.2\%) & 218 (39.6\%) & 0.03 \\
\hline
\end{tabular}

A very good state of health was declared mostly by students from families with high FAS, compared to students from low FAS families. Pneumonia and bronchitis affected mostly 
Table 3. Health self-assessment, Family Affluence Scale (FAS) and health problems vs. parents' education

\begin{tabular}{|c|c|c|c|c|c|c|c|c|}
\hline & \multicolumn{4}{|c|}{ Mother's education } & \multicolumn{4}{|c|}{ Father's education } \\
\hline & $\mathrm{N}$ & Primary or vocational school & High school or university & $p$ value & $\mathrm{N}$ & Primary or vocational school & High school or university & $p$ value \\
\hline \multicolumn{9}{|c|}{ Health self-assessment } \\
\hline Average or poor & \multirow{3}{*}{1067} & $54(16.9 \%)$ & $126(16.8 \%)$ & \multirow{3}{*}{ NS } & \multirow{3}{*}{1046} & $55(15.1 \%)$ & $123(18.1 \%)$ & \multirow{3}{*}{ NS } \\
\hline Rather good & & $155(48.6 \%)$ & $379(50.7 \%)$ & & & $191(52.3 \%)$ & $328(48.2 \%)$ & \\
\hline Very good & & $110(34.5 \%)$ & $243(32.5 \%)$ & & & $119(32.6 \%)$ & $230(33.8 \%)$ & \\
\hline \multicolumn{9}{|l|}{ FAS scale } \\
\hline Low & \multirow{3}{*}{1054} & $102(32.2 \%)$ & 117 (15.9\%) & \multirow{3}{*}{$\mathrm{p}<0001$} & \multirow{3}{*}{1035} & $89(24.7 \%)$ & $122(18.1 \%)$ & \multirow{3}{*}{$<0.01$} \\
\hline Average & & $145(45.7 \%)$ & $271(36.8 \%)$ & & & $157(43.5 \%)$ & $253(37.5 \%)$ & \\
\hline High & & $70(22.1 \%)$ & $349(47.4 \%)$ & & & $115(31.7 \%)$ & 299 (44.4\%) & \\
\hline Asthma & 1040 & $25(7.9 \%)$ & $78(10.8 \%)$ & NS & 1019 & $31(8.7 \%)$ & $71(10.7 \%)$ & NS \\
\hline Pneumonia & 1059 & $104(32.9 \%)$ & $270(36.3 \%)$ & NS & 1039 & $111(30.6 \%)$ & $255(37.7 \%)$ & 0.02 \\
\hline Bronchitis & 1062 & $144(45.4 \%)$ & 349 (46.9\%) & NS & 1042 & $160(44.2 \%)$ & $323(47.5 \%)$ & NS \\
\hline Spinal deformities & 1059 & 109 (34.1\%) & $298(40.3 \%)$ & $\mathrm{p}=0.05$ & 1037 & $131(36.2 \%)$ & $273(40.4 \%)$ & NS \\
\hline
\end{tabular}

NS- not significant

students from families with an average FAS. Bronchial asthma was the least common in students from families with medium FAS, while the highest asthma prevalence was observed in the group of teenagers from families with low FAS. Spinal deformities were very rare among young people characterized by high FAS. Young people living in a troubled neighbourhood more frequently suffered from bronchial asthma, pneumonia and spinal deformities; in addition, they self-assessed their health as low. Only bronchitis more often concerned students from the average neighbourhoods, in comparison with those from more troubled residential areas.

The education level of students' parents was also taken into consideration. This indicator was analyzed to understand its relationship with students' health conditions, health selfassessment and FAS (Tab. 3).

It was statistically significant that students whose parents had primary or vocational education declared the lowest level of family affluence. Spinal deformities significantly more often affected young people whose mothers had completed at least high school. Pneumonia occurred more often among students from families where the fathers were high school, college or university graduates. The differences among those groups were statistically significant.

\section{DISCUSSION}

Inequalities in health have been and for a long time will be for Public Health [1-3]. Medical science approach towards public health, which started at the turn of $19^{\text {th }}$ and $20^{\text {th }}$ centuries, focused mainly on increasing the significance of socio-economic markers. At the same time, public health in the USA and UK became a public issue in close correlation with the Industrial revolution [11]. The main focus was on vastly increasing risk of diseases among the poorest of the populastion. The main objective of Public Health became the improvement of this condition. At first, the plan seemed to be successful, but very soon it became only an illulusion: existing health inequalities were not reduced, and at some level they became even more severe. There was, and still is, no direct correlation between public wealth and socio-economical improvements, and the diminution of health inequalities which concern even the richest countries in the world. For example, in Canada, the UK and USA, the percentage of children living below the socio-economic minimum essential standard ranges between $15 \%-28 \%$ [12].

Therefore, bearing the above in mind, it was fully justified to survey the lower secondary school students in Bytom and discover more about their state of health. The issue of health inequality needed to be analysed taking into account its macro-social context.

A very important aspect that should not be omitted is the development and transformation of post-industrial cities such as Bytom. In the second half of the $19^{\text {th }}$ century, Bytom was one of the most important centres of German heavy industry [13]. At that time, the city was growing very fast in all possible ways: economically, socially and in terms of its cultural resources. In the period after World War II, the city did not experience significant investments, and consequently suffered from industrial deterioration. This was caused mostly by the fact that the German population was largely expelled by the Polish and Soviet armies, and re-populated with expatriate Poles from the eastern provinces annexed by the Soviet Union. Some of the indigenous Silesian population remained, but the sense of local community disappeared. Moreover, uncontrolled and sudden development of heavy industry during the post-war Communist era in Poland had a devastating effect on Bytom. The turbulent transition from Communism to Democracy in Poland had an impact on socio-economic position of the population, and the negative results of transition - economic polarization and pauperization of society - were responsible for the growth of health inequalities [14].

The transformation also revealed very complex challenges for the city, both socio-economic and demographical: industry, oriented to one branch, became practically non-existent, which contributed to a rapid increase in unemployment, the threat of dysfunctions and social marginalization, resulting in the formation of a so-called underclass. This is the part of the population that occupies the lowest possible position in a class hierarchy and forms 'ghettos'. They are therefore experiencing multidimensional deprivation - social, cultural and economic, and are often referred to as 'lost in transition'; however, this group of 
people is not a marginal underclass because its number have been increasing. Families of industrial workers have been particularly affected by the poverty resulting from the fall of industry and loss of income [14].

Accumulation of a community's problems determines the state of local health, both objectively and subjectively. In this study, most of the students described their health as good. A characteristic fact was that the interviewed boys statistically self-assessed their health as better than girls. Similar observations were made in a cross-national HBSC study in 2010. In all analysed countries the interviewed girls statistically self-assessed their health as worse than that of boys [15], a result that is partly explained by puberty during which girls aged 11-15 experience hormonal changes, which become the cause of their greater emotional lability [16].

Results of the presented study clearly show that the accumulation of problems in the place of residence has a significant effect on pneumonia, bronchitis, spinal deformities and health self-assessment. A number of similar studies have also highlighted the fact that poverty and poor quality of living have a negative effect on the state of health of children and teenagers [17]. Children living in neighbourhood with a high intensity of problems are more exposed to other risks, including injuries, accidents or environmental pollution; they have reduced access to sports and cultural infrastructure, and they also tend to adopt unhealthy behaviour patterns [17]. In the current study, the predominance of students declaring a high intensity of problems in their place of residence can be observed, especially in comparison with the cross-national HBSC study from 2010 [9]. In another cross-national study, the percentage of teenagers living in a places with a high intensity of problems ranged from $31.2 \%-36.6 \%$, while in the presented study it was $51 \%$. This is not the first time that such a discrepancy has been discovered, and it is not specific only to the Bytom area. In earlier surveys, up to $44 \%$ of secondary school students from the Silesian Province indicated a high level of different types of issues in their place of residence [18]. This fact can be explained and understood in a broader context when taking into consideration the above-mentioned fast growth of industrialised cities. It is worth mentioning that national studies indicate that the scale which was implemented in this study has a great level of suitability $[9,18]$.

Less obvious are the correlations between the specific health markers and families' affluence measured by FAS scale, a probable reason being low reliability of the FAS scale and the selected material markers describing the families' affluence. The lack of own room, a small number of computer in the home, lack of a car, and scarcity of trips outside the place of residence do not always characterize familie with a low financial status. Numerous studies have proved that the FAS scale, in fact, could be very useful, especially in terms of analysing health inequalities $[9,18]$. Even with all its imperfections, the scale is still described as the most objective SES marker in studying teenagers $[9,18]$. Students aged 13-16 are often unable to determine the level of economic status of their families [19]. The FAS scale is more useful in assessing material status than direct questions concerning family finances [18].

The discrepancies in the prevalence of pneumonia, asthma and bronchitis among children from low and high FAS families were not statistically significant. Only the family affluence was a strong determinant of young people's health self-assessment. Moreover, spinal deformities more often affected children from families with low income than high FAS level families; these discrepancies, however, were not statistically significant. Nevertheless, the results were worth mentioning. According to Altman et al., the 'absence of evidence is not evidence of absence'[20].

There are a number of reasons why in the current study spinal deformities more often affected children from families with low income than high FAS level families, for example, little knowledge about the possible risks for children's health, lower access to a specialist when it comes to medical care, but mostly material and economic deprivation. While discussing the spinal deformities, a hypothesis seems plausible, that the worse the quality of the environment, including local environment, the higher the health risks, resulting inter alia from the absence of a sports and cultural infrastructure. The results of this study clearly show that there was an association between spinal deformities and the quality of the residential area: the lower the quality of living, the more common the occurrence of spinal deformities. Therefore, the relationship between family affluence, place of residence and state of health is clearly observed.

On the contrary, a detailed analysis of relationship between the education of parents and the children's state of health suggests that the above proposal is not justified. Spinal deformities were more often among teenagers whose parents had higher (high school, college, university) level of education. On the one hand, this can be explained by increased awareness, and related to more frequent medical examinations. In line with parents' education, family affluence increases and undoubtedly has a significant effect, enabling students to use the sport infrastructure and actively spend their free time. On the other hand, the differences can also be explained by the great diversity of the population inhabiting different city districts. Bytom city is not homogenous and also has more prestige districts. However, as a result of the negligence in all the city areas (architecture, infrastructure), even the more prestigious districts are inhabited by both poor and wealthy families. In addition, more than a half of the interviewed students mentioned that their residential areas could be called 'troubled'.

A limitation of this study is the low participation rate, but paradoxically, the low participation rate results from attention to ethical issues of the research. It was assumed that students, as minors, could not decide about their participation in the survey and parents' permission is needed. This is why a letter explaining the reason for and scope of this study, as well as a declaration of consent to complete the questionnaire, was given to parents via the children. It is also probable that some students did not pass the letter to their parents or return the letter signed by the parents, which must have had an impact on the participation rate.

The presented study concerns an extremely important topic, not only from the point of view public health, and in a sense, it was a novelty in Silesia. The assessment of health status of young people living in Bytom, with regard to the socio-economic factors, can be used by local government to allocate funds and make decisions for planning prevention programmes and health education. Although the HBSC surveys undertaken in Poland also concerned the Silesian Province, the current study fully covers the characteristics of the population and subjective assessment of health based on health indicators. 
Health inequalities constitute a very extensive subject which concerns many issues, but analyses always need to refer to social and cultural contexts, in which individuals and groups function.

\section{CONCLUSIONS}

A high level of family affluence and living in an area with little intensification of socio- economic problems have an influence on students' health self-assessment marked as good or very good. It is necessary to introduce more effective health promoting activities in order to lessen the differences in the state of health of teenagers living in Bytom, and in the rest of the country and Europe. The scale describing the relationship between the troubled living areas and the assessment of socio-economic markers is very useful in describing the state of health of Bytom's lower secondary school students. Regarding the FAS scale, the results are less obvious and a direct relationship between material markers of families and state of health markers cannot be established unequivocally.

\section{REFERENCES}

1. WHO. Constitution of the World Health Organization Conference, New York: 1946.(http://apps.who.int/gb/bd/PDF/bd47/EN/constitution-en. pdf, accessed 14.07.2014)

2. Commission of the European Communities. White Paper. Together for Health A Strategic Approach for the EU 2008-2013. Brussels, 2007.

3. Whitehead M. The concepts and principles of equality and health. World Health Organisation, Copenhagen, 1990.

4. Robinson LM, Mcintyre L,Officer S. Welfare babies:poor children's experiences informing healthy peer relationships in Canada. Health Promot Int. 2005; 4: 342-350

5. Central Statistical Office. Department of Social and Living Condition Studies. European study on theincome and the living conditions (EUSILC) in 2010r [in Polish]. Warsaw, 2011.

6. Central Statistical Office. Employment, wages and salaries in national economy in Ist half of 2012. Statistical Information and Elaborations. Warsaw, 2012
7. Municipality of Bytom. Office of Town Development. Report on the Bytom town state 2009. Bytom, 2010.

8. Maniecka-Bryła I, Pikala M, Bryła M. Health inequalities among rural and urban inhabitants of Łódź Province, Poland. AnnAgric Environ Med. 2012; 19(4): 723-731

9. Mazur J, Małkowska-Szkutnik A. The HBSC studyresults 2010. Technical report [in Polish]. Warsaw, 2011

10. Mazur J, Woynarowska B. Measures of the social inequalities in questionnaire studies on school youth [in Polish]. PrzeglEpidemiol 2004; 58 (2): 377-90.

11. Berkman LF, Kawachi I. A historical framework for social epidemiology. In Berkman LF, Kawachi I (eds). Social epidemiology. Oxford University Press; New York 2000.pp 3-12.

12. Wilkinson R, Marmot M. Social determinants of health. The solid facts. Second edition. WHO, Denmark 2003. (http://www.euro.who. int/_data/assets/pdf_file/0005/98438/e81384.pdf, accessed 14.07.2014)

13. Official service of Bytom. Some words on Bytom history. [inPolish] (http://www.bytom.pl/pl/9/1197155410/1197128419/4, accessed 14.07.2014)

14. Piątkowski W, Skrzypek M. Utilization of non-medical healing methods as a way of coping with life difficulties in the socially deprived 'losers' of the systemic transformation processes in Poland. AnnAgric Environ Med2012, 19(1): 147-15

15. Currie C, Zanotti C, Morgan A, Currie D, de Looze M, Roberts C. et al. (eds). Social determinants of health and well-being among young people. Health Behaviour in School-aged Children (HBSC) study: international report from the 2009/2010 survey.WHO Regional Office for Europe, Copenhagen 2012 (accesed 14.07.2014http://www.hbsc.org/ publications/international/)

16. Gådin KG, Hammarström A. A possible contributor to the higher degree of girls reporting psychological symptoms compared with boys in grade nine? Eur J Pub Health 2005; 15(4):380-385.

17. Mathieson A, Nemer L. Socio-environmentally determined health inequities among children and adolescents. Summary of outcomes and country case studies. WHO, Regional Office for Europe. Copenhagen 2010.

18. Mazur J. Impact of the financial status of the family and the environment on the lifestyle of youth aged 15 years. The HBSC study results environmental approach. Warsaw 2007 [in Polish].

19. Goodman, E. The role of socioeconomic status gradients in explaining differences in US adolescents' health. Am J Public Health 1999; 89 (10):1522-8

20. Altman DG, Bland M.Absence of evidence is not evidence of absence. Br Med J 1995; 311: 485 